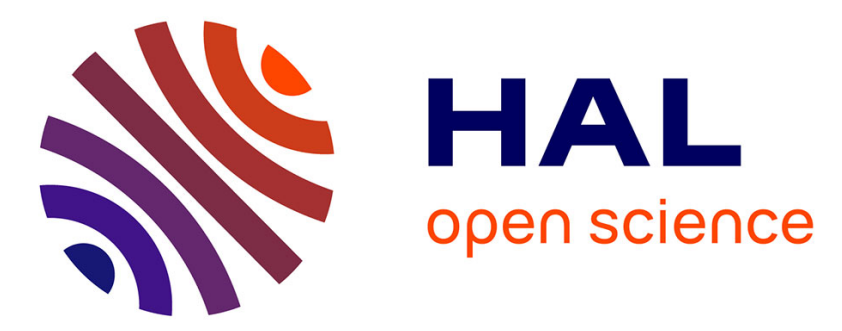

\title{
Les ménages arbitrent-ils entre coût du logement et coût du transport: une réponse dans le cas francilien
}

Nicolas Coulombel, Fabien Leurent

\section{To cite this version:}

Nicolas Coulombel, Fabien Leurent. Les ménages arbitrent-ils entre coût du logement et coût du transport: une réponse dans le cas francilien. Economie et Statistique / Economics and Statistics, 2013, 457-458, pp.57-75. hal-00926248

\section{HAL Id: hal-00926248 \\ https://hal.science/hal-00926248}

Submitted on 9 Jan 2014

HAL is a multi-disciplinary open access archive for the deposit and dissemination of scientific research documents, whether they are published or not. The documents may come from teaching and research institutions in France or abroad, or from public or private research centers.
L'archive ouverte pluridisciplinaire HAL, est destinée au dépôt et à la diffusion de documents scientifiques de niveau recherche, publiés ou non, émanant des établissements d'enseignement et de recherche français ou étrangers, des laboratoires publics ou privés. 


\title{
Les ménages arbitrent-ils entre coût du logement et coût du transport : une réponse dans le cas francilien
}

\author{
Nicolas Coulombel et Fabien Leurent ${ }^{*}$
}

\begin{abstract}
En Île-de-France, le niveau élevé des prix immobiliers se traduit par une part du revenu consacrée au logement plus grande qu'en province. Dans ce contexte, la hausse du prix des carburants pourrait peser sur la solvabilité des ménages. Plusieurs auteurs soutiennent que ce risque est accentué dans les zones périurbaines et rurales. L'éloignement de Paris induirait des coûts de transport importants venant s'ajouter à un budget logement déjà élevé. Or le modèle monocentrique de l'économie urbaine vient sinon contredire, du moins nuancer cette affirmation. Ce modèle prédit une fongibilité des budgets transport et logement, de partielle à totale selon les hypothèses retenues. Les coûts de transport élevés dans les zones excentrées seraient donc compensés par un plus faible budget logement.
\end{abstract}

Cette étude teste les prédictions du modèle monocentrique concernant la fongibilité des budgets logement et transport dans le cas des ménages franciliens. Les informations présentes dans 1'Enquête Globale de Transport 2001-02 permettent de développer un test économétrique simple (significativité de la variable de coût de transport dans l'équation de dépense de logement), mais inédit à ce jour.

A statut d'occupation donné, le revenu constitue le principal déterminant du budget logement, les autres caractéristiques du ménage ayant un impact secondaire. Les différentes variables de coût de transport testées sont non significatives ou d'influence marginale. En particulier, l'élasticité du budget logement au budget transport est nulle pour les ménages accédants à la propriété et locataires du secteur privé, et faiblement positive $(0,025)$ dans le secteur social. Ces résultats amènent à rejeter l'hypothèse d'un arbitrage entre coût du logement et coût du transport. De manière annexe, l'étude trouve un lien positif entre dépense de logement et prix au $\mathrm{m}^{2}$. Ceci remet en question le choix fréquent de fonctions d'utilité Cobb-Douglas pour modéliser les préférences résidentielles des ménages.

\footnotetext{
* Université Paris-Est, Laboratoire Ville Mobilité Transport, UMR T9403 (ENPC IFSTTAR UPEMLV), 6 et 8 av. Blaise Pascal, Cité Descartes F-77455 Marne-la-Vallée Cedex 2.

Les auteurs remercient les trois rapporteurs anonymes, Jean-Paul Hubert et Didier Blanchet pour leurs précieux conseils, notamment sur une précédente version de l'article. La qualité de l'article s'en est trouvée fortement améliorée. Ils souhaitent également remercier Marion Deschamps pour sa contribution à la préparation de nombreuses données.
} 


\section{Introduction}

Le logement représente une dépense de plus en plus lourde pour les ménages français. En 2010, ils consacraient en moyenne $22 \%$ de leur revenu disponible brut aux dépenses courantes de logement (déduction faite des aides personnelles), contre 18\% en 1990 (CGDD, 2012). Dans ce contexte, la hausse conjointe des prix des carburants ${ }^{1}$ fait peser un risque accru sur la solvabilité des ménages. Ce risque est particulièrement prégnant en Île-de-France. D'une part, le niveau des prix immobiliers y induit des taux d'effort logement (part du revenu consacrée à ce poste) plus élevés qu'en province (Gallot et al., 2011). D'autre part, la flambée des prix dans le centre de l'agglomération pousse de nombreux ménages à s'éloigner de Paris faute de pouvoir accéder au logement de la taille désirée. Or s'installer en périphérie pourrait in fine s'avérer plus coûteux, comme le soutiennent Hare (1995) aux Etats-Unis ou Polacchini et Orfeuil (1999) concernant l'Île-de-France. Les fortes dépenses de transport associées aux styles de vie périurbain et rural aboutiraient à un budget global logement-transport plus élevé que dans les zones centrales. Les ménages franciliens éloignés, au premier rang desquels les moins aisés, seraient donc les plus vulnérables à la hausse des coûts de transport.

Cette thèse va à l'encontre d'une autre thèse largement répandue d'une fongibilité des dépenses de logement et de transport. Cette idée a été popularisée par le modèle de Wingo. Celui-ci établit que sous certaines hypothèses (exposées plus loin), la somme du budget logement et du budget transport est une constante, et donc indépendante de la localisation (Fujita, 1989). Partant d'hypothèses moins fortes, le modèle monocentrique de l'économie urbaine prédit également une fongibilité des deux budgets, mais partielle. Au final, le haut niveau des coûts de transport en franges des aires urbaines ne serait donc pas problématique, puisque intégré par les ménages et le marché immobilier.

Cet article vise à éclairer ce débat en testant la fongibilité des dépenses de logement et de transport au niveau désagrégé (du ménage). Nous estimons par régression linéaire multivariée l'influence du budget transport sur le budget logement des ménages franciliens, mesuré par le loyer hors charge ou la mensualité du (ou des) prêt(s) immobilier(s). D'autres variables de coût de transport sont également testées : la distance domicile-travail et le budget temps quotidien du chef de ménage, ainsi que la zone IAURIF de résidence.

A statut d'occupation donné, le revenu constitue le principal déterminant du budget logement. Les autres caractéristiques du ménage telles que sa taille ou l'âge du chef de ménage ont une influence secondaire. Les variables transport sont non significatives ou d'influence marginale. En particulier, l'élasticité du budget logement au budget transport est nulle pour les ménages accédants à la propriété et les ménages locataires du secteur privé. Elle est faiblement positive $(0,025)$ pour ceux bénéficiant d'un logement social, ce qui pourrait refléter les contraintes portant sur le choix du logement dans ce secteur. Ces résultats amènent à rejeter l'hypothèse d'un arbitrage entre coût du logement et coût du transport. Le choix de l'éloignement est bien associé à des prix au $\mathrm{m}^{2}$ plus faibles ainsi qu'à des surfaces plus grandes, mais pas à une dépense de logement plus faible. Par rapport aux prédictions de notre modèle théorique, les ménages éloignés surconsommeraient donc du logement. De manière annexe, nous trouvons un lien positif entre la dépense de logement et le prix au $\mathrm{m}^{2}$. Ce point remet en question le choix répandu de fonctions d'utilité de type Cobb-Douglas pour modéliser les préférences résidentielles des ménages.

\footnotetext{
${ }^{1}$ Sur la même période, le prix de l'essence sans plomb 95 augmentait de 0,80€ à 1,30€.
} 
L'Enquête Globale de Transport 2001-2002 constitue la base de données principale pour notre analyse. Cette enquête étudie la mobilité des ménages franciliens tout en fournissant divers éléments sur leurs conditions de logement, dont le montant de la dépense de logement. Nous pouvons ainsi développer une approche économétrique étudiant le lien entre les budgets transport et logement au niveau du ménage. Cet élément constitue un apport majeur par rapport aux travaux précédents, reposant uniquement sur des valeurs moyennes par zone. Nous proposons également une méthodologie plus robuste pour estimer les différents coûts de transport (argent, distance et temps) à partir de données de mobilité, basée sur l'utilisation de modèles de réseau.

\section{Liens entre dépenses de logement et de transport : revue de la littérature}

Au sein du vaste corpus étudiant les liens entre logement et transport, trois thèmes ont fait l'objet d'une attention spécifique : ${ }^{2}$

- les liens entre mobilité résidentielle et mobilité quotidienne (dont l'influence du temps de trajet domicile-travail sur la décision de déménager) ;

- la place du transport dans les stratégies résidentielles des ménages;

- l'impact de l'offre de transports sur les prix immobiliers.

Le modèle monocentrique joue un rôle central dans cette littérature. Sa popularité doit à ce qu'il offre un cadre d'analyse simple mais puissant pour étudier les deux dernières questions. La question des liens entre budgets logement et transport se situant à la frontière de ces sujets, le modèle monocentrique s'avère particulièrement pertinent. Nous allons donc commencer par en rappeler les principaux résultats relatifs à notre sujet.

Dans le modèle monocentrique standard tel qu'exposé par Fujita (1989), tous les emplois sont concentrés en un point central de la ville, dénommé Central Business District (ou CBD). Chaque ménage se rend au CBD pour y travailler, ce qui lui procure un revenu $Y$. Ce revenu lui permet de couvrir ses frais de transport et de consommer deux biens : un bien logement, mesuré par $s$, et un bien composite, mesuré par $z$, pris comme numéraire et représentant l'ensemble des autres biens dans l'économie. Les ménages sont supposés rationnels et maximisent une fonction d'utilité $U(z, s)$ sous contrainte de budget :

$$
\max _{z, s, r} U(z, s) \text { s.c. } R(r) s+z+T(r)=Y
$$

où $r$ représente la distance au $\mathrm{CBD}, R(r)$ la rente foncière, et $T(r)$ le coût de transport lié à la migration pendulaire. On fait l'hypothèse supplémentaire que $T(r)$ augmente strictement avec la distance au centre.

Comme s'éloigner du CBD entraîne un surcoût au niveau du transport, in fine les ménages arbitrent entre l'accessibilité du lieu et le niveau des prix immobiliers (respectivement mesurés par la distance au centre $r$ et la rente foncière $R(r)$ ). L'intérêt du modèle réside dans le fait que les prix soient déterminés de manière endogène. A l'équilibre concurrentiel du marché immobilier, la rente foncière capitalise ainsi l'avantage comparatif de localisation : $R(r)$ décrôit avec $r$.

Quelle conséquence pour les budgets logement et transport? Premièrement, nous pouvons observer que le programme de maximisation peut se réécrire sous la forme :

$$
\max _{z, s, r} U(z, s) \text { s.c. } R(r) s+z=Y-T(r)
$$

\footnotetext{
${ }^{2}$ Le lecteur pourra se référer à Gobillon (2001) et Coulombel (2010) pour des revues de la littérature sur ces thèmes.
} 
A localisation donnée, le ménage choisit $z$ et $s$ (et par suite son budget logement) en fonction du revenu net $Y-T(r)$ et non du revenu total $Y$.

Supposons en premier lieu que la taille des logements soit constante et égale à $s_{0}$. Le modèle prédit alors une parfaite fongibilité des budgets logement et transport : $R(r) s_{0}+T(r)=C^{t e}$. Pour les ménages éloignés, le surcoût en transport est parfaitement compensé par l'économie sur la dépense de logement, via le mécanisme de capitalisation des prix immobiliers.

Quand les ménages choisissent librement la taille du logement, celle-ci augmente strictement en s'éloignant du CBD tandis que $z$ diminue. Par conséquent, la somme des dépenses de logement et transport $R(r) s+T(r)$ n'est plus constante mais augmente strictement avec $r$. Dans le cas général, on ne peut en revanche rien en déduire concernant l'influence de $r$ sur le budget logement : $R(r)$ décroissant et $s$ croissant avec $r$, il y a indétermination. Coulombel (2010) démontre que si la fonction d'utilité vérifie une certaine condition de régularité, le budget logement décroît strictement avec $r .{ }^{3}$ Ceci est le cas pour les fonctions de type CobbDouglas. Lorsque $(z, s)=s^{\alpha} z^{1-\alpha}$, la dépense de logement vaut : $R(r) s=\alpha(Y-T(r))$. Cette quantité décroît bien strictement avec $r$.

En conclusion, si la fonction d'utilité $U(z, s)$ vérifie la fonction de régularité évoquée, le modèle monocentrique prédit que le budget logement décroît strictement avec $r$, ou - cela est équivalent - avec $T(r)$. Plus précisément, l'élasticité du budget logement au budget transport est strictement négative, inférieure à -1 , et égale à -1 uniquement si la taille des logements est constante. Le modèle prédit également que le budget logement dépend uniquement du revenu net $Y-T(r)$, et non du revenu total $Y$.

A notre connaissance, les prédictions du modèle monocentrique évoquées, ou plus généralement l'hypothèse d'un arbitrage entre coût de logement et coût de transport, n'ont pas encore fait l'objet de tests économétriques. Plusieurs travaux basés sur une approche agrégée offrent néanmoins de premiers éléments de réponse, notamment pour l'île-de-France. Polacchini et Orfeuil (1999) étudient ainsi comment les taux d'effort transport et logement des ménages franciliens varient selon leur lieu de résidence et leur statut d'occupation. Les coûts de transport sont calculés à partir de l'Enquête Globale de Transport 1991-92. Les dépenses de logement proviennent de l'Observatoire des Loyers de l'Agglomération Parisienne, qui fournit des moyennes par zone pour un découpage de la région en neuf zones de prix. Comme dans cet article, la dépense de logement est définie comme le loyer dans le cas de ménages locataires ou les mensualités d'emprunt(s) dans le cas d'accédants à la propriété. Les auteurs observent que le taux d'effort logement varie peu entre les zones, tandis que le taux d'effort transport augmente dans les zones éloignées (qui sont également les moins chères). Au final, les auteurs trouvent une certaine fongibilité des budgets transport et logement dans le cœur de l'agglomération, mais pas dans les zones éloignées. L'hypothèse d'un arbitrage entre les deux budgets serait donc partiellement invalidée. L'étude comporte néanmoins plusieurs limitations importantes. En premier lieu, les budgets sont obtenus à partir de deux sources différentes. Ceci restreint l'analyse à des comparaisons de moyennes par zone, sans validation économétrique des résultats. L'étude ne prend également pas en compte le niveau de revenu, alors que celui-ci est, nous le verrons, un déterminant majeur du taux d'effort logement.

Berri (2007) étudie la même question à partir des Enquêtes Budget des Familles de 1978-79, 84-85, 89 et 94-95. L'auteur trouve pour l'ensemble de la période des résultats similaires : le taux d'effort logement varie peu selon la zone de résidence, tandis que le taux d'effort transport croît avec l'éloignement de Paris. Berri en parvient à remettre en cause l'hypothèse

\footnotetext{
${ }^{3}$ Cette condition est : $\forall s>0,-Z_{s s}(s, u) s>Z_{s}(s, u)$, où $Z(s, u)$ est la fonction inverse de $U$ selon $s$.
} 
d'arbitrage entre coût de logement et coût de transport, au moins pour les ménages de revenu modeste. Les ménages de revenu élevé seraient plus à même d'arbitrer entre les deux. Ceci constitue d'ailleurs un des apports de l'étude, qui prend partiellement en compte le revenu via des terciles de niveau de vie. Cependant, aucune approche économétrique n'est menée et l'auteur ne contrôle qu'une variable à la fois (tercile de revenu ou statut d'occupation).

Aux Etats-Unis, Haas et al. (2006) parviennent aux mêmes résultats dans une étude semblable portant sur 28 métropoles américaines. Le taux d'effort logement varie peu au sein de chaque métropole, tandis que le taux d'effort transport augmente fortement en s'éloignant des pôles d'emploi. Le taux d'effort logement décroît également fortement avec le niveau de revenu. Encore une fois, l'étude suit une approche agrégée en comparant des moyennes par zone de recensement (census tract), sans fournir de confirmation économétrique au niveau du ménage.

\section{Présentation de l'aire d'étude et du zonage IAURIF}

La région Île-de-France présente de fortes disparités spatiales du point de vue du marché du logement comme de celui du système de transport. Considérant cette difficulté et la question posée ici, le zonage IAURIF offre en première approche une grille de lecture pertinente. La région est découpé en huit zones homogènes du point de vue de l'offre de transports collectifs en particulier (annexe, Graphique II). Cette première propriété est importante, le budget transport étant, nous le verrons, fortement lié au niveau d'équipement automobile du ménage et au mode de transport principalement utilisé. Le zonage se caractérise également par une augmentation progressive de la distance moyenne au centre de Paris, allant de $3 \mathrm{~km}$ pour la première zone à $53 \mathrm{~km}$ pour la dernière. Cette deuxième propriété nous permettra de confronter nos premières analyses aux prédictions du modèle monocentrique, l'aire urbaine de Paris étant elle-même relativement monocentrique (Coulombel, 2010, p.220).

Concernant le logement, le zonage IAURIF reflète assez fidèlement l'évolution spatiale de la structure des marchés locaux. Les deux premières zones, et dans une moindre mesure les Villes Nouvelles, présentent un marché tourné vers la location et le logement collectif. A Paris comme en banlieue intérieure, près de $60 \%$ des ménages vivent dans le secteur locatif et plus de $80 \%$ dans un logement collectif (Tableau 1). Le parc des franges de l'agglomération et des deux dernières zones est à l'inverse axé vers la propriété (avec de 66\% de ménages propriétaires pour les villes secondaires isolées à $90 \%$ pour les communes rurales) et le logement individuel (représentant de 60\% du parc pour les franges de l'agglomération à 96\% pour les communes rurales).

Ce zonage est en revanche hétérogène du point de vue des prix immobiliers. Il ne tient notamment pas compte de la forte dissymétrie est-ouest (Coulombel, 2010, p.221). ${ }^{4}$ Le fait de mieux refléter l'offre de transports collectifs que le niveau des prix immobiliers constituera, nous le verrons, une dernière propriété désirable du zonage IAURIF. Nous nous appuierons donc sur celui-ci pour une première analyse basée sur des moyennes par zone. Il servira également, mais dans une moindre mesure, lors de la modélisation économétrique.

Nous pouvons noter encore trois points concernant l'aire d'étude. Le revenu moyen varie modérément entre les zones, allant de $2222 €$ pour les villes secondaires isolées à $2924 €$ pour les communes rurales (Tableau 1). La taille des ménages varie plus fortement en s'éloignant de Paris : de 1,88 personnes en moyenne à Paris, à 2,83 dans les communes rurales. Enfin, les trois premières zones concentrent la large majorité $(70 \%)$ de la population francilienne.

\footnotetext{
${ }^{4}$ Berri (2007) a étudié l'influence de redécouper le zonage selon l'axe est/ouest sur les résultats. Il parvient aux mêmes résultats qu'avec le zonage non découpé.
} 
Tableau 1

Caractéristiques générales de la population francilienne, 2001

\begin{tabular}{|l|c|c|c|cccc|c|}
\hline Zone IAURIF & Population & $\begin{array}{c}\text { Taille } \\
\text { moyenne } \\
\text { du ménage }\end{array}$ & $\begin{array}{c}\text { Revenu } \\
\text { mensuel } \\
\text { moyen }(€)\end{array}$ & $\begin{array}{c}\text { Locatif } \\
\text { privé }\end{array}$ & $\begin{array}{c}\text { Locatif } \\
\text { social }\end{array}$ & Accédants & $\begin{array}{c}\text { Propriétaires } \\
\text { \& Locataires } \\
\text { à titre gratuit }\end{array}$ & $\begin{array}{c}\% \\
\text { logement } \\
\text { individuel }\end{array}$ \\
\hline Paris & 2143069 & 1,88 & 2657 & $43 \%$ & $17 \%$ & $5 \%$ & $35 \%$ & $0.3 \%$ \\
Banlieue intérieure & 3006568 & 2,40 & 2385 & $27 \%$ & $30 \%$ & $9 \%$ & $33 \%$ & $17 \%$ \\
Banlieue extérieure & 2664252 & 2,57 & 2482 & $19 \%$ & $24 \%$ & $16 \%$ & $41 \%$ & $39 \%$ \\
$\begin{array}{l}\text { Franges de } \\
\text { l'agglomération }\end{array}$ & 908017 & 2,74 & 2923 & $14 \%$ & $17 \%$ & $24 \%$ & $46 \%$ & $60 \%$ \\
Villes Nouvelles & 815098 & 2,87 & 2404 & $14 \%$ & $33 \%$ & $26 \%$ & $28 \%$ & $45 \%$ \\
Villes secondaires & 828847 & 2,68 & 2256 & $14 \%$ & $27 \%$ & $23 \%$ & $35 \%$ & $54 \%$ \\
axes/vallées & 279003 & 2,62 & 2222 & $14 \%$ & $20 \%$ & $15 \%$ & $51 \%$ & $72 \%$ \\
Villes secondaires & 421723 & 2,83 & 2924 & $8 \%$ & $2 \%$ & $28 \%$ & $62 \%$ & $96 \%$ \\
isolées & 11066577 & 2,40 & 2518 & $26 \%$ & $23 \%$ & $13 \%$ & $37 \%$ & $29 \%$ \\
\hline Communes rurales & Ensemble & $2,4 \%$ & & \\
\hline
\end{tabular}

Lecture: en 2001, Paris comptait 2,1 millions habitants selon l'EGT. Un ménage comptait 1,88 membres en moyenne, pour un revenu mensuel de 2657 euros. 17\% des ménages résidaient dans le secteur social, et 0,3\% dans un logement individuel. Champ : tous ménages (sauf colonne revenu : ménages dont le revenu est renseigné).

Source : Enquête Globale de Transport 2001-2002, DREIF

\section{Quelle mesure du budget logement choisir?}

L'Enquête Globale de Transport (EGT) est la principale enquête portant sur la mobilité quotidienne des ménages franciliens ( $c f$. Encadré 1). Elle fournit également de nombreuses informations sur les conditions de logement des ménages enquêtés, en particulier leur dépense de logement depuis l'édition 2001-2002. C'est sur cette édition que nous nous appuierons.

Le budget logement peut se mesurer de plusieurs manières. Le loyer constitue la mesure de référence chez les locataires, éventuellement augmenté des charges locatives voire d'autres charges récurrentes (assurance, taxes, travaux d'entretien...). Pour les propriétaires occupants, deux mesures coexistent: les mensualités liées au remboursement du ou des prêts (qui ne concernent que les ménages accédants) et le loyer imputé. ${ }^{5}$ Cet article étudie les arbitrages entre coût de logement et coût de transport, en lien notamment avec la question de la solvabilité des ménages. La dépense mensuelle effective (i.e. les mensualités des prêts) constitue donc la mesure plus appropriée.

L'EGT 2001-2002 fournit le loyer mensuel hors charges pour les ménages locataires et la mensualité totale (en cas de plusieurs emprunts) pour les accédants à la propriété. Nous retiendrons cette définition pour le budget logement. Les charges telles que définies dans le Compte du Logement (incluant sept postes : entretien et petits travaux des parties communes, petits travaux dans le logement, multirisques-habitation, frais de personnel, prestations de service, déménagements, frais et baux d'agences) ne sont pas comptabilisées. Ces charges ont cependant un poids modeste. En 2001, elles s'élevaient à 19 milliards d'euros, contre 150 milliards d'euros de loyers (DAEI/SES-P et DGUHC, 2007). Nous ferons donc l'hypothèse que les charges ne modifient pas les arbitrages étudiés ici. De même, notre choix de nous concentrer sur les arbitrages de court-moyen terme tels que mesurés par les dépenses mensuelles effectives justifie de ne pas considérer l'apport initial dans le cas des accédants. ${ }^{6}$

\footnotetext{
${ }^{5}$ Le loyer imputé correspond au loyer de marché du logement occupé par le ménage propriétaire. Il peut être considéré comme un loyer fictif que le ménage se verse à lui-même.

${ }^{6}$ Sur la période 1998-2002, le taux d'apport personnel moyen est de 39,3\% en agglomération parisienne (Daubresse, 2003).
} 
L'approche retenue exclut de facto deux catégories de ménages de l'analyse:

- les locataires à titre gratuit (3\% des ménages franciliens d'après l'EGT 2001-2002) ;

- les propriétaires de plein droit (33\% des ménages).

Pour ces deux catégories, le budget logement est nul selon la définition retenue. Cela ne signifie pas que ces ménages n'arbitrent pas entre budget transport et budget logement. Les locataires à titre gratuit font le choix de vivre dans leur logement. Ils pourraient chercher dans le secteur locatif un autre logement si leur logement actuel les soumettait à une dépense de transport trop importante. Nous n'étudierons pas cette hypothèse, par ailleurs peu probable au vu des ordres de grandeur respectifs des budgets logement et transport ( $c f$. infra).

Dans le cas des ménages propriétaires de plein droit, l'arbitrage peut avoir eu lieu par le passé si le ménage était préalablement accédant. L'arbitrage pourrait également se situer sur une autre échelle de temps, celle du long terme. Pour tester cette hypothèse, la mesure pertinente pour les propriétaires occupants dans leur ensemble ne serait plus les remboursements de prêt mais le coût d'opportunité de vivre dans le logement détenu, c'est-à-dire le loyer imputé. Malgré la complémentarité avec le sujet de cet article, nous ne chercherons pas à tester cette hypothèse, au vu notamment des importantes difficultés méthodologiques liées à l'estimation des loyers imputés (voir notamment Driant et Jacquot, 2005, à ce sujet).

Encadré 1

\section{Présentation de l'Enquête Globale de Transport}

L'Enquête Globale de Transport, diffusée par la DRIEA Île-de-France (ex-DREIF), est la principale enquête portant sur la mobilité des ménages franciliens. La procédure d'enquête repose sur l'utilisation de deux formulaires, un pour les jours de semaine, l'autre pour les fins de semaine. Le premier interroge tous les déplacements effectués par le ménage pour un jour donné, ce jour variant d'un ménage à l'autre. Le second fait de même, mais pour l'ensemble du week-end (samedi et dimanche) et de manière moins détaillée.

La base est structurée en quatre niveaux de données, avec les principales variables suivantes:

- ménage : lieu de résidence, composition, revenu, équipement en véhicules (automobiles, utilitaires, motocycles, cycles), caractéristiques du logement, dépense de logement.

- individu : âge, activité, CSP, lieu de travail, abonnement TC.

- déplacement : point d'origine et de destination (codés selon un carroyage de $300 \mathrm{~m}$ de côté), motif du déplacement, mode de transport principal utilisé, heure de départ et d'arrivée, conditions de stationnement.

- moyen (maillon élémentaire du déplacement reflétant les changements de mode lors du déplacement) : carreau d'origine et de destination, mode et titre de transport utilisé.

Lors de l'édition 2001-2002, 10478 ménages ont répondu à l'enquête jour de semaine, pour un total de 23656 individus et un taux d'échantillonnage de $0,23 \%$. Un tiers des ménages a également répondu au formulaire fin de semaine. L'échantillon utilisé dans ce travail a été obtenu en filtrant les catégories de ménage suivantes :

- propriétaires de plein droit et locataires à titre gratuit (cf. supra) ;

- ménage dont le revenu ou la dépense de logement n'est pas renseigné ;

- premier sextile de revenu ( $c f$. infra). 
Ces opérations réduisent notre échantillon à 4893 ménages, dont la composition est présentée en Annexe,

Tableau 6.

Limites des données de coût de logement

L'édition 2001-2002 est la première à intégrer des questions portant sur le coût du logement, mesuré par le niveau du loyer hors charges ou le montant total des remboursements d'emprunts pour les ménages accédants. Elle est sujette à diverses limites méthodologiques. Dans le cas de ménages bénéficiant d'aides au logement, il n'a pas été précisé si les aides ont été déduites du montant indiqué, ni leur propre montant. Une comparaison avec l'Enquête Logement 2002 (Omalek, 2003) met en évidence des taux d'effort logement plus élevés dans l'EGT, notamment pour les ménages modestes. Nous servant de la même Enquête Logement, nous trouvons que le différentiel entre le taux d'effort brut et le taux d'effort net des aides de logement décroît fortement avec le niveau de revenu (Annexe, Tableau 7). Ces éléments tendent à indiquer que les aides n'auraient en général pas été prises en compte dans l'EGT, d'où des taux d'effort plus élevés. Afin de limiter tout biais potentiel dans nos résultats, nous excluons de l'échantillon le premier sextile de revenu, pour lequel le différentiel entre taux d'effort brut et net est significatif. A l'inverse, le différentiel est minime pour les ménages ayant un revenu supérieur au revenu médian (avec moins de 2 points d'écart en moyenne).

\section{La dépense moyenne de logement varie peu selon la zone de résidence}

Le budget logement moyen d'un ménage francilien croît avec le niveau de revenu, ce quel que soit le statut d'occupation et la zone IAURIF considérés (Tableau 2). Une seule exception est observée pour le parc social des villes secondaires isolées : un ménage du tercile médian y consacrait en moyenne $304 €$ à son logement, contre $310 €$ pour un ménage du $2^{\text {ème }}$ sextile.

Les ménages bénéficiant d'un logement social présentent logiquement des budgets inférieurs aux autres ménages. L'écart est particulièrement marqué pour les hauts niveaux de revenu : en 2001, un ménage du tercile supérieur dépensait en moyenne $404 €$ par mois pour résider en banlieue extérieure dans le parc social, contre $776 €$ pour le parc locatif privé et $881 €$ pour les accédants. La différence entre accédants et locataires du secteur privé est moins prononcée.

En revanche, le budget moyen varie peu entre les zones IAURIF. Pour le tercile médian, le loyer moyen dans le secteur privé se situe ainsi entre $441 €$ (villes secondaires isolées) et $579 €$ (franges de l'agglomération).

Tableau 2

Budget logement mensuel moyen selon la zone de résidence (en $€$ )

\begin{tabular}{|l|c|c|c|c|c|c|c|c|c|}
\hline \multirow{2}{*}{ Zone IAURIF } & \multicolumn{3}{|c|}{ Accédants } & \multicolumn{3}{c|}{ Locatif Social } & \multicolumn{3}{c|}{ Locatif Privé } \\
\cline { 2 - 10 } & $\begin{array}{c}\text { 2ème } \\
\text { Sextile }\end{array}$ & $\begin{array}{c}\text { Tercile } \\
\text { Médian }\end{array}$ & $\begin{array}{c}\text { Tercile } \\
\text { Sup. }\end{array}$ & $\begin{array}{c}\text { 2ème } \\
\text { Sextile }\end{array}$ & $\begin{array}{c}\text { Tercile } \\
\text { Médian }\end{array}$ & $\begin{array}{c}\text { Tercile } \\
\text { Sup. }\end{array}$ & $\begin{array}{c}\text { 2ème } \\
\text { Sextile }\end{array}$ & $\begin{array}{c}\text { Tercile } \\
\text { Médian }\end{array}$ & $\begin{array}{c}\text { Tercile } \\
\text { Sup. }\end{array}$ \\
\hline Paris & 378 & 485 & 1008 & 310 & 406 & 541 & 428 & 526 & 903 \\
Banlieue intérieure & 394 & 583 & 925 & 306 & 376 & 441 & 389 & 492 & 745 \\
Banlieue extérieure & 455 & 535 & 881 & 319 & 370 & 404 & 375 & 485 & 776 \\
Franges de l'agglomération & 444 & 632 & 870 & 335 & 415 & 417 & 386 & 579 & 731
\end{tabular}




\begin{tabular}{|l|c|c|c|c|c|c|c|c|c|} 
Villes Nouvelles & 389 & 637 & 862 & 354 & 428 & 459 & 446 & 512 & 674 \\
Villes secondaires & 346 & 602 & 854 & 343 & 397 & 464 & 416 & 511 & 650 \\
axes/vallées & - & 604 & 705 & 310 & 304 & 404 & 361 & 441 & 849 \\
Villes secondaires isolées & 361 & 478 & 850 & 388 & 446 & 488 & 365 & 525 & 770 \\
Communes rurales & 405 & 577 & 891 & 319 & 388 & 458 & 403 & 508 & 813 \\
\hline Ensemble &
\end{tabular}

Lecture: en 2001, un ménage appartenant au tercile de revenu supérieur et résidant à Paris dans le secteur locatif privé consacrait en moyenne $903 €$ par mois à son logement.

Champ : locataires et accédants (sauf premier sextile de revenu et revenu/budget logement non renseigné)

Source : Enquête Globale de Transport 2001-2002, DREIF

L'analyse des taux d'effort logement donne lieu à des observations similaires concernant le statut d'occupation et la zone de résidence (Tableau 3). En revanche, le taux d'effort moyen décroît fortement avec le niveau de revenu (comme précédemment observé par Berri, 2007). Dans le secteur locatif privé, il diminue de $35 \%$ pour le $2^{\text {ème }}$ sextile à $20 \%$ pour le tercile supérieur.

Tableau 3

Taux d'effort logement moyen selon la zone de résidence

\begin{tabular}{|c|c|c|c|c|c|c|c|c|c|}
\hline \multirow[b]{2}{*}{ Zone IAURIF } & \multicolumn{3}{|c|}{ Accédants } & \multicolumn{3}{|c|}{ Locatif social } & \multicolumn{3}{|c|}{ Locatif privé } \\
\hline & $\begin{array}{l}\text { 2ème } \\
\text { Sextile }\end{array}$ & $\begin{array}{l}\text { Tercile } \\
\text { Médian } \\
\end{array}$ & $\begin{array}{l}\text { Tercile } \\
\text { Sup. }\end{array}$ & $\begin{array}{l}\text { 2ème } \\
\text { Sextile }\end{array}$ & $\begin{array}{l}\text { Tercile } \\
\text { Médian } \\
\end{array}$ & $\begin{array}{l}\text { Tercile } \\
\text { Sup. }\end{array}$ & $\begin{array}{l}\text { 2ème } \\
\text { Sextile }\end{array}$ & \begin{tabular}{|l} 
Tercile \\
Médian \\
\end{tabular} & $\begin{array}{l}\text { Tercile } \\
\text { Sup. }\end{array}$ \\
\hline Paris & $31 \%$ & $24 \%$ & $22 \%$ & $28 \%$ & $21 \%$ & $15 \%$ & $38 \%$ & $27 \%$ & $21 \%$ \\
\hline Banlieue intérieure & $33 \%$ & $28 \%$ & $22 \%$ & $27 \%$ & $19 \%$ & $13 \%$ & $35 \%$ & $25 \%$ & $18 \%$ \\
\hline Banlieue extérieure & $37 \%$ & $26 \%$ & $21 \%$ & $28 \%$ & $19 \%$ & $12 \%$ & $32 \%$ & $24 \%$ & $20 \%$ \\
\hline Franges de l'agglomération & $36 \%$ & $30 \%$ & $21 \%$ & $30 \%$ & $21 \%$ & $12 \%$ & $34 \%$ & $29 \%$ & $19 \%$ \\
\hline Villes Nouvelles & $33 \%$ & $30 \%$ & $22 \%$ & $32 \%$ & $22 \%$ & $15 \%$ & $40 \%$ & $26 \%$ & $20 \%$ \\
\hline $\begin{array}{l}\text { Villes secondaires } \\
\text { axes/vallées }\end{array}$ & $29 \%$ & $30 \%$ & $22 \%$ & $31 \%$ & $20 \%$ & $15 \%$ & $35 \%$ & $27 \%$ & $19 \%$ \\
\hline Villes secondaires isolées & - & $29 \%$ & $20 \%$ & $28 \%$ & $15 \%$ & $14 \%$ & $30 \%$ & $22 \%$ & $19 \%$ \\
\hline Communes rurales & $27 \%$ & $24 \%$ & $21 \%$ & $35 \%$ & $25 \%$ & $13 \%$ & $34 \%$ & $27 \%$ & $23 \%$ \\
\hline Ensemble & $33 \%$ & $28 \%$ & $22 \%$ & $28 \%$ & $20 \%$ & $13 \%$ & $35 \%$ & $26 \%$ & $20 \%$ \\
\hline
\end{tabular}

Lecture: en 2001, un ménage appartenant au tercile de revenu supérieur et résidant à Paris dans le secteur locatif privé consacrait en moyenne $21 \%$ de son revenu au budget logement.

Champ : locataires et accédants à la propriété (sauf premier sextile de revenu et revenu/budget logement non renseigné)

Source : Enquête Globale de Transport 2001-2002, DREIF

\section{Quelle mesure du budget transport choisir?}

Le budget transport couvre l'ensemble des dépenses d'un ménage liées aux déplacements des individus qui le composent. Ces dépenses sont de deux natures : celles liées à l'utilisation des transports collectifs (TC), comprenant l'achat d'abonnements et/ou de tickets, et celles liées à l'utilisation de véhicules particuliers (VP). Ces dernières prennent la forme de coûts fixes et de coûts variables, auxquels s'ajoute les coûts de stationnement. La méthodologie de calcul est détaillée en Encadré 2.

Le champ est restreint aux dépenses liées à la mobilité quotidienne. La mobilité vacancière, par ailleurs non couverte par l'EGT, est exclue de l'analyse. Si des liens existent entre ces deux types de mobilité, ce choix est conforme à notre modèle théorique. Dans celui-ci, le coût du transport correspond à une dépense récurrente et contrainte, liée à la migration pendulaire. 
Les dépenses de mobilité vacancière sont donc à comptabiliser dans $z$ (le bien composite), et non dans le budget transport.

Encadré 2

\section{Méthodologie de calcul des dépenses de transport}

Le budget transport mensuel d'un ménage possède deux composantes : le poste transports collectifs (TC) et le poste véhicules particuliers (VP).

Le budget TC est calculé à partir du type de titre de transport utilisé pour chaque déplacement. Dans le cas d'un abonnement, le coût mensuel (tenant compte du zonage) est imputé en tenant compte le cas échéant de la participation de l'employeur.

Le budget VP distingue coûts fixes et coûts variables. Les coûts variables (carburant, entretien du véhicule et achat d'accessoires) sont estimés à partir de la mobilité quotidienne observée. Les coûts fixes (achat du véhicule, assurance) sont alloués de manière forfaitaire par type de véhicule (VP, 2 roues motorisé ou vélo) possédé, au prorata de la mobilité quotidienne sur la mobilité totale (mesurées en kms parcourus par an).

Pour être plus précis, deux cas se présentent concernant le calcul des coûts variables. Si le ménage a répondu aux deux questionnaires - jour de semaine et week-end -, nous sommons les coûts obtenus pour le jour de semaine et pour la fin de semaine, en pondérant par le nombre de jours adéquat. Les jours fériés et les vacances sont considérés comme des jours de fin de semaine. Si le ménage n'a pas répondu au questionnaire fin de semaine, la méthodologie est similaire exception faite de ce que le budget coût variable week-end est imputé, en tenant compte du type de ménage, de sa localisation et de sa motorisation.

\section{Utilisation de sources complémentaires portant sur les prix/coûts des transports}

L'Enquête Globale de Transport ne renseignant pas directement les coûts monétaires liés aux déplacements, d'autres sources ont dû être mobilisées.

Concernant les transports en commun, le document technique produit par Garcia Castello (2006) fournit les prix pour les tickets « $\mathrm{t} »$ (métro et bus) et pour les abonnements en 2001. L'équation de prix pour les autres tickets (trains et RER) est due à Agenais (2008).

Concernant les véhicules particuliers, les coûts fixes sont estimés via l'utilisation de l'EGT pour déterminer le parc de véhicules du ménage (voitures, motos, vélos,..) et de l'Enquête Budget des Familles (Cérani et Camus, 2004) pour estimer le coût fixe moyen par type de véhicule. Le calcul des coûts variables repose sur le modèle d'affectation routier pour estimer les consommations de carburant ( $c f$. Encadré 3$)$ combiné à diverses sources donnant :

- les prix des divers carburants (Observatoire de l'Énergie, 2003);

- un ratio reliant le budget carburant aux autres coûts variables (Cérani et Camus, 2004).

Enfin, le coût du stationnement tient compte du type (sur voirie, parking payant, tarif résident, etc.) et du lieu de stationnement, ainsi que de sa durée en cas de stationnement payant.

En toute rigueur, la mobilité quotidienne non contrainte devrait également être exclue du champ de l'analyse. Contrairement à la mobilité vacancière, ce point soulève deux difficultés: savoir définir la mobilité non contrainte, et surtout pouvoir mesurer les dépenses afférentes. L'importance du phénomène de boucles de déplacement en Île-de-France (Leurent et Samadzad, 2010) rend notamment complexe l'imputation des coûts par motif de déplacement. Pour illustrer ce point, prenons le cas d'un individu allant travailler. Sur le chemin de retour, il fait un détour pour aller au cinéma, puis rentre chez lui. Dans ce schéma, on voit la difficulté de distinguer au sein de la dépense totale de transport ce qui est lié au trajet domicile-travail et ce qui est lié à la mobilité de loisir, surtout que l'arrêt au cinéma pourrait influer sur le choix 
de mode (et donc sur la dépense). Face à cette difficulté, nous utiliserons la variable distance domicile-travail du chef de ménage comme proxy du budget «mobilité contrainte » et testerons également cette variable lors de l'analyse économétrique.

En plus de la dépense, nous calculons deux coûts de transport non monétaires : le temps de transport total sur une journée et la distance parcourue correspondante. Contrairement aux approches précédentes reposant sur des données déclarées (Polacchini et Orfeuil, 1999), les budgets temps et les distances parcourues sont calculés via l'utilisation conjointe de l'EGT et de modèles de réseau ( $c f$. Encadré 3). Cette démarche vise à disposer de distances plus réalistes (distance réseau et non à vol d'oiseau éventuellement corrigée d'un facteur de courbure) et de temps de parcours plus «robustes » dans le sens où contrairement aux données déclarées, ils ne sont affectés ni par l'occurrence d'aléas divers le jour de l'enquête (accidents, travaux, etc.), ni par les erreurs de saisie ou de déclaration.

Les dépenses sont calculées au niveau du ménage sur une base mensuelle. Les budgets non monétaires sont calculés au niveau individuel et pour un jour de semaine. ${ }^{7}$

Encadré 3

\section{Reconstruire les déplacements à partir de modèles d'affectation routier et TC}

Les modèles d'affectation (ou modèles de réseau) simulent les comportements de choix d'itinéraire des individus étant donné les conditions de circulation sur un réseau (routier ou de transports collectifs). Ils permettent de déterminer l'usage des différentes infrastructures de transport ou missions dans le cas des TC, à l'équilibre du trafic.

A l'aide d'un modèle de réseau, il est possible de connaître le chemin minimisant le temps de parcours entre un point d'origine et un point de destination, appelé Plus Court Chemin (PCC). Le modèle fournit également la longueur du PCC, et donc la vitesse moyenne.

\section{Présentation des modèles utilisés}

Deux modèles de réseau sont utilisés : un modèle routier et un modèle TC. Tous deux sont basés sur des modèles de la DRIEA Île-de-France calés à l'aide de l'EGT 2001-02, convertis pour pouvoir opérer sous le logiciel TransCAD. Nos modèles routier et TC comportent respectivement 28478 et 56163 arcs. Les chemins choisis par les individus minimisent le temps de parcours entre l'origine et la destination, tenant compte de la congestion dans le cas routier, et des temps d'attente, de correspondance et de marche dans le cas des TC.

Une comparaison a été effectuée entre données déclarées et résultats des modèles. Le budget temps moyen pour un jour de semaine est de 74 minutes en déclaratif, contre 64 minutes avec notre méthodologie. L'écart peut s'expliquer par des erreurs des deux côtés : les vitesses sont probablement légèrement surestimées dans le modèle d'affectation routier, inversement l'EGT contient de nombreux temps de parcours déclarés aberrants (aboutissant par exemple à des vitesses inférieures à $1 \mathrm{~km} / \mathrm{h}$ en voiture).

\section{Estimation des consommations de carburant}

En combinant les modèles d'affectation routier et de consommation COPERT3, il est possible d'estimer pour un déplacement donné la consommation de carburant associée, suivant le type et la puissance du véhicule utilisé (l'estimation tenant compte du type de carburant).

\footnotetext{
${ }^{7}$ Ce dernier choix traduit l'hypothèse que la mobilité de semaine est plus régulière, et donc plus prévisible, que celle de fin de semaine, et par là mieux à même d'être prise en compte lors du choix de localisation résidentielle.
} 


\section{Le budget transport moyen croît fortement en s'éloignant de Paris}

En 2001, le budget transport mensuel moyen d'un ménage francilien s'élevait à $228 €$ (annexe, Tableau 8). Il varie fortement selon la zone de résidence, la dépense croissant en s'éloignant de Paris (Graphique I). Le budget varie ainsi d'un facteur 1 à 2 suivant que l'on réside à Paris ou en périphérie. Pour les ménages résidant en zone rurale, la dépense atteint même $531 €$ par mois en moyenne, soit plus du triple des Parisiens (151€). Ces éléments tendent à soutenir l'hypothèse monocentrique de notre modèle théorique, le coût de transport moyen augmentant avec la distance au centre de l'agglomération. ${ }^{8}$

Deux éléments supplémentaires se dégagent de l'analyse spatiale des budgets transport. D'une part, la voiture pèse très fortement dans le budget transport des ménages franciliens. Le poste «Véhicules Particuliers » prédomine dans toutes les zones, représentant de 70 à $90 \%$ du budget moyen (annexe, Tableau 8). Le zonage IAURIF est donc particulièrement pertinent, offrant des niveaux de service homogènes pour les transports en commun.

Deuxièmement, la hausse du budget moyen de transport du centre vers la périphérie est portée quasi-intégralement par le poste «Véhicules Particuliers ». De $106 €$ à Paris, celui-ci atteint $485 €$ dans les communes rurales. Le niveau élevé du poste VP en périphérie résulte en premier lieu d'une plus forte motorisation des ménages périurbains. Ceux-ci possèdent 3 à 4 fois plus de voitures que les ménages résidant à Paris (annexe, Tableau 8). Cette plus forte motorisation a comme effet d'accroître mécaniquement le poste de coûts fixes lié au VP, et influe également sur les coûts variables via un recours accru à la voiture. Pour autant, elle n'explique pas toute la hausse. Les coûts variables progressent en effet plus vite que les coûts fixes, jusqu'à les dépasser en niveau pour les deux dernières zones (annexe, Tableau 8).

\section{Graphique I}

\section{Budget transport mensuel moyen selon la zone de résidence}

\footnotetext{
${ }^{8}$ La relation n'est pas parfaite : le budget moyen est plus élevé dans les franges de l'agglomération (322€) que dans les Villes Nouvelles (296€). Cette irrégularité tient à la faible offre de TC dans les franges par rapport aux Villes Nouvelles, bien desservies par le RER, et aboutissant à un taux de motorisation plus élevé que dans les zones IAURIF contigües.
} 


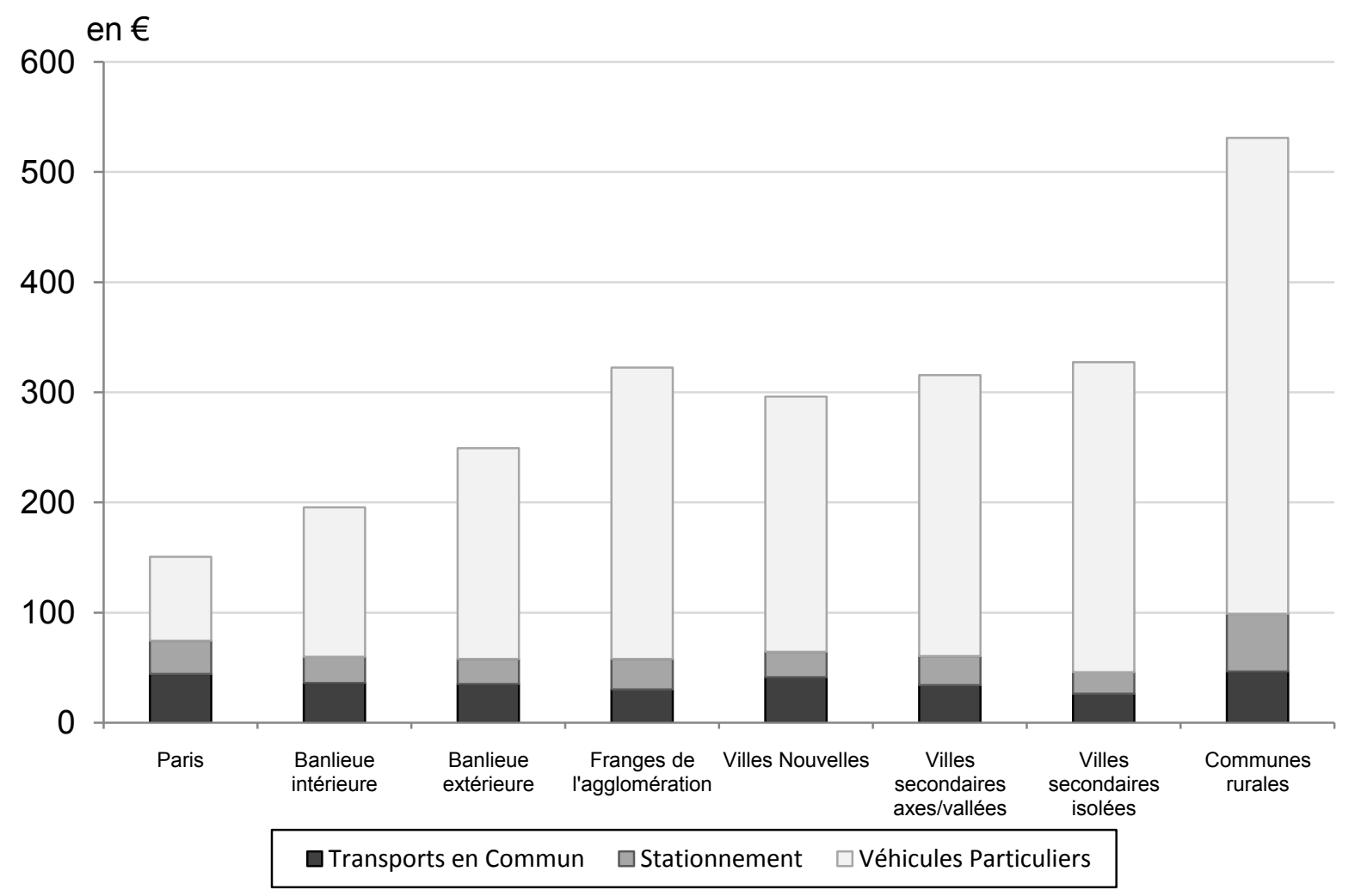

Champ : locataires et accédants à la propriété (sauf premier sextile de revenu et revenu/budget logement non renseigné) Source : Enquête Globale de Transport 2001-2002, DREIF

Le second facteur contribuant au niveau élevé des coûts de transport dans les zones excentrées est l'augmentation des distances parcourues (annexe, Graphique III). Ce point concerne essentiellement les ménages motorisés. Un individu dont le ménage dispose d'une voiture parcourait ainsi en moyenne $18 \mathrm{~km}$ un jour de semaine s'il résidait à Paris, contre $48 \mathrm{~km}$ en zone rurale. Cette hausse des distances parcourues n'est pour autant pas transmise aux budgets temps, qui varient peu selon les zones ainsi que selon le niveau de motorisation (annexe, Graphique IV).

Le poste «Transports en Commun» est lui relativement stable quelle que soit la zone de résidence. Si le recours accru à la voiture concorde avec un nombre réduit d'usagers des transports collectifs dans les zones éloignées, ceux-ci disposent d'abonnements plus chers en moyenne (car couvrant plus de zones), d'où la relative stabilité observée.

Le taux d'effort transport moyen croît également de manière soutenue en s'éloignant de Paris (Tableau 4). Il dépasse fréquemment $15 \%$ dans les dernières zones, contre de 5 à $8 \%$ à Paris en moyenne selon les catégories de ménage considérées. Si le taux d'effort décroît du tercile médian au tercile supérieur pour la quasi-totalité des localisations et statuts d'occupation, cette observation ne se vérifie pas pour le 2ème sextile et le tercile médian. Les taux d'effort semblent également peu varier entre statuts d'occupation.

Tableau 4

Taux d'effort transport moyen selon la zone de résidence

\begin{tabular}{|c|c|c|c|c|c|c|c|c|c|}
\hline \multirow{2}{*}{ Zone IAURIF } & \multicolumn{3}{|c|}{ Accédants } & \multicolumn{3}{c|}{ Locatif social } & \multicolumn{3}{c|}{ Locatif privé } \\
\cline { 2 - 8 } & $\begin{array}{c}\text { 2ème } \\
\text { Sextile }\end{array}$ & $\begin{array}{c}\text { Tercile } \\
\text { Médian }\end{array}$ & $\begin{array}{c}\text { Tercile } \\
\text { Sup. }\end{array}$ & $\begin{array}{c}\text { 2ème } \\
\text { Sextile }\end{array}$ & $\begin{array}{c}\text { Tercile } \\
\text { Médian }\end{array}$ & $\begin{array}{c}\text { Tercile } \\
\text { Sup. }\end{array}$ & $\begin{array}{c}\text { 2ème } \\
\text { Sextile }\end{array}$ & $\begin{array}{c}\text { Tercile } \\
\text { Médian }\end{array}$ & $\begin{array}{c}\text { Tercile } \\
\text { Sup. }\end{array}$ \\
\hline
\end{tabular}




\begin{tabular}{|l|c|c|c|c|c|c|c|c|c|}
\hline Paris & $5 \%$ & $5 \%$ & $7 \%$ & $8 \%$ & $8 \%$ & $6 \%$ & $7 \%$ & $6 \%$ & $6 \%$ \\
Banlieue intérieure & $12 \%$ & $11 \%$ & $8 \%$ & $9 \%$ & $11 \%$ & $9 \%$ & $10 \%$ & $10 \%$ & $7 \%$ \\
Banlieue extérieure & $20 \%$ & $14 \%$ & $10 \%$ & $12 \%$ & $12 \%$ & $10 \%$ & $12 \%$ & $11 \%$ & $9 \%$ \\
Franges de l'agglomération & $17 \%$ & $16 \%$ & $11 \%$ & $12 \%$ & $13 \%$ & $10 \%$ & $15 \%$ & $14 \%$ & $9 \%$ \\
Villes Nouvelles & $15 \%$ & $16 \%$ & $10 \%$ & $13 \%$ & $14 \%$ & $13 \%$ & $11 \%$ & $15 \%$ & $9 \%$ \\
Villes secondaires & $17 \%$ & $18 \%$ & $13 \%$ & $16 \%$ & $16 \%$ & $11 \%$ & $14 \%$ & $15 \%$ & $13 \%$ \\
axes/vallées & - & $23 \%$ & $13 \%$ & $16 \%$ & $19 \%$ & $21 \%$ & $24 \%$ & $15 \%$ & $11 \%$ \\
Villes secondaires isolées & $40 \%$ & $25 \%$ & $16 \%$ & $19 \%$ & $44 \%$ & $11 \%$ & $26 \%$ & $16 \%$ & $16 \%$ \\
\hline
\end{tabular}

Lecture: en 2001, un ménage appartenant au tercile de revenu supérieur et résidant à Paris dans le secteur locatif privé consacrait en moyenne $6 \%$ de son revenu aux dépenses de transport (liées à la mobilité quotidienne).

Champ : locataires et accédants à la propriété (sauf premier sextile de revenu et revenu/budget logement non renseigné)

Source : Enquête Globale de Transport 2001-2002, DREIF

\section{Le coût de transport n'influe pas sur la dépense de logement}

Nos premiers résultats ne corroborent pas l'hypothèse d'un arbitrage entre budget logement et budget transport. Comparé aux ménages résidant en cœur d'agglomération, les ménages éloignés présentent des taux d'effort transport plus élevés en moyenne pour des taux d'effort logement relativement similaires.

Pour confirmer ce résultat, nous étudions les déterminants du budget logement par régression linéaire multivariée sous le logiciel SAS, et testons la significativité de différentes variables liées au coût de transport. Les estimations sont stratifiées par statut d'occupation. Le choix du statut d'occupation pourrait en effet être lié à des caractéristiques inobservées du ménage, telles que l'importance accordée à la propriété ou au logement en général. Dans la mesure où ces caractéristiques pourraient également influer sur le niveau du budget logement, une estimation non stratifiée souffrirait d'un problème d'endogénéité. La solution idéale face à cette difficulté est de modéliser simultanément le choix du statut d'occupation et l'équation de dépense de logement. Lorsque, comme ici, les données ne permettent pas l'estimation d'un tel modèle, l'estimation stratifiée fournit néanmoins des élasticités conditionnelles très proches de celles obtenues par l'estimation jointe (Henderson et Ioannides, 1986; Goodman, 1990).

La variable dépendante est le budget logement auquel nous appliquons la transformation de Box-Cox. Le paramètre $\lambda$ est déterminé à l'aide de la macro Box-Cox de SAS, qui indique une transformation optimale pour $\lambda=0$ (correspondant à la transformée logarithmique). Les variables explicatives incluent uniquement des caractéristiques du ménage et la variable transport testée. Aucune caractéristique du logement ou du voisinage n'est prise en compte, le choix du logement étant endogène au choix du budget logement. Enfin, cinq variables liées au coût de transport sont testées séparément (donnant donc lieu à cinq modèles différents) :

1. le logarithme du budget transport $\log (T(r))$;

2. le budget transport transformé sous la forme $\log (Y-T(r))-\log (Y)$;

3. la distance domicile-travail du chef de ménage ;

4. le budget temps de transport du chef de ménage (pris pour un jour de semaine) ;

5. la zone IAURIF de résidence.

Le modèle 1 fournit l'élasticité du budget logement au budget transport conditionnelle au statut d'occupation. La transformation du budget transport (modèle 2) dérive de notre modèle théorique, selon lequel les ménages se basent sur leur revenu disponible $Y-T(r)$ et non sur le revenu total $Y$ pour effectuer leurs choix de consommation. Sous cette hypothèse, les 
coefficients des variables explicatives $\log (Y)$ et $\log (Y-T(r))-\log (Y)$ doivent être égaux. Les modèles 1 et 2 offrent deux tests alternatifs des prédictions du modèle monocentrique. Les modèles 3 à 5 permettent de confirmer nos résultats en considérant d'autres mesures alternatives du coût de transport ainsi qu'un effet de localisation.

Afin de faciliter la lecture, seul le modèle 1 est présenté en détail. Pour les autres modèles, nous reportons uniquement les coefficients des variables transport, les autres coefficients et les $\mathrm{R}^{2}$ ne variant que marginalement par rapport au modèle 1 . L'hypothèse d'homoscédasticité des résidus n'est rejetée au seuil de $5 \%$ pour aucun modèle.

Le revenu constitue le déterminant majeur du budget logement (Tableau 5). Pour chaque statut d'occupation, nous avons pu vérifier que la somme des carrés de type II associée à la variable revenu était largement supérieure à celles associées aux autres variables, avec un facteur 20 en moyenne. Nous trouvons une élasticité revenu du budget logement de 0,54 dans le secteur locatif privé, 0,53 pour les ménages accédants, et 0,23 dans le secteur locatif social. Ceci correspond pour les mêmes secteurs à des élasticités revenu du taux d'effort logement de respectivement $-0,46,-0,47$ et $-0,77$. Le taux d'effort logement décroît fortement avec le niveau de revenu du ménage, en particulier dans le secteur locatif social.

Les coefficients des variables liées à la taille du ménage présentent le signe attendu, la dépense de logement augmentant avec le nombre de membres. Les écarts sont le plus marqués dans le secteur locatif privé. Un ménage de 4 personnes y présente un budget logement en moyenne $17 \%$ plus élevé qu'un ménage de 2 personnes.

L'ancienneté dans le logement est inversement associée à une dépense plus faible. Ceci peut s'expliquer par le fait qu'une fois installé, le ménage voit en moyenne son revenu progresser plus vite que sa dépense de logement. Cette dernière ne varie notamment pas dans le temps pour les accédants (les mensualités étant fixes). Pour les locataires, l'encadrement de la hausse des loyers joue certainement un rôle important.

L'âge du chef de ménage influe principalement dans le secteur locatif privé : les chefs de ménage âgés présentent des taux d'effort logement plus élevés $(+10 \%$ pour les 50 à 64 ans, $+14 \%$ pour les plus de 65 ans) que les chefs de ménage plus jeunes. Une interprétation possible est que les ménages âgés conservent souvent le logement qu'ils occupaient avec leurs enfants, ou gardent dans tous les cas le goût de logements spacieux. Leur désir de surface accru se traduirait donc, toutes choses égales par ailleurs, par une dépense plus élevée.

\section{Tableau 5}

Effets de différents facteurs sur la dépense de logement

\begin{tabular}{|c|c|c|c|c|c|c|}
\hline & \multicolumn{2}{|c|}{ Locatif privé } & \multicolumn{2}{|c|}{ Accédants } & \multicolumn{2}{|c|}{ Locatif social } \\
\hline & Paramètre & $\begin{array}{l}\text { Ecart- } \\
\text { type }\end{array}$ & Paramètre & Ecart-type & Paramètre & Ecart-type \\
\hline Constante & $2,21^{* * *}$ & 0,13 & $2,17^{* \star \star}$ & 0,19 & $4,02^{* * *}$ & 0,16 \\
\hline Logarithme du revenu mensuel (en $€$ ) & $0,53^{* * *}$ & 0,017 & $0,54^{\star * *}$ & 0,023 & $0,23^{* * *}$ & 0,022 \\
\hline $\begin{array}{l}\text { Taille du ménage (en personnes) } \\
1 \\
2\end{array}$ & $\begin{array}{r}-0,086^{* * *} \\
R\end{array}$ & 0,024 & $\begin{array}{r}-0,091^{* *} \\
R\end{array}$ & ef. & $\begin{array}{r}-0,032 \\
R\end{array}$ & fef. \\
\hline $\begin{array}{l}3 \\
4 \\
5 \text { ou plus }\end{array}$ & $\begin{array}{l}0,091^{* * *} \\
0,16^{* * *} \\
0,14^{* * *}\end{array}$ & $\begin{array}{l}0,026 \\
0,030 \\
0,039\end{array}$ & $\begin{array}{c}0,039 \\
0,080^{* *} \\
0,12^{* * *}\end{array}$ & $\begin{array}{l}0,032 \\
0,032 \\
0,036\end{array}$ & $\begin{array}{c}0,061^{* *} \\
0,071^{* * *} \\
0,11^{* * *}\end{array}$ & $\begin{array}{l}0,025 \\
0,027 \\
0,028\end{array}$ \\
\hline $\begin{array}{l}\text { Age du chef de ménage } \\
30 \text { ans ou moins } \\
31 \text { à } 49 \text { ans }\end{array}$ & $\begin{array}{r}-0,0097 \\
R\end{array}$ & 0,023 & $\begin{array}{r}-0,079 * \\
R\end{array}$ & óf. & $\begin{array}{r}-0,016 \\
R\end{array}$ & o, o, \\
\hline
\end{tabular}




\begin{tabular}{|c|c|c|c|c|c|c|}
\hline $\begin{array}{l}50 \text { à } 64 \text { ans } \\
65 \text { ans et plus }\end{array}$ & $\begin{array}{c}0,099^{* * *} \\
0,13^{* * *}\end{array}$ & $\begin{array}{l}0,025 \\
0,034\end{array}$ & $\begin{array}{l}-0,023 \\
0,0018\end{array}$ & $\begin{array}{l}0,027 \\
0,080\end{array}$ & $\begin{array}{c}0,059^{\star \star *} \\
0,046\end{array}$ & $\begin{array}{l}0,021 \\
0,029\end{array}$ \\
\hline $\begin{array}{l}\text { Durée d'occupation du logement } \\
\text { Nombre d'années (si inférieur à } 10 \text { ans) } \\
\text { Indicatrice durée > à } 10 \text { ans }\end{array}$ & $\begin{array}{l}-0,012^{* * *} \\
-0,18^{\star * *}\end{array}$ & $\begin{array}{c}0,0041 \\
0,028\end{array}$ & $\begin{array}{l}-0,014^{* * *} \\
-0,17^{* * *}\end{array}$ & $\begin{array}{c}0,0043 \\
0,031\end{array}$ & $\begin{array}{l}-0,011^{* * *} \\
-0,13^{* * *}\end{array}$ & $\begin{array}{c}0,0041 \\
0,026\end{array}$ \\
\hline $\begin{array}{l}\text { Autres caractéristiques } \\
\text { Chef de ménage inactif (autre que } \\
\text { retraité) } \\
\text { Chef de ménage de sexe féminin } \\
\text { Couple bi-actif occupé }\end{array}$ & $\begin{array}{c}0,068^{*} \\
0,070^{* * *} \\
-0,084^{* * *}\end{array}$ & $\begin{array}{l}0,038 \\
0,022 \\
0,023 \\
\end{array}$ & $\begin{array}{c}0,036 \\
-0,019 \\
0,028 \\
\end{array}$ & $\begin{array}{l}0,071 \\
0,037 \\
0,025 \\
\end{array}$ & $\begin{array}{c}-0,077^{* * *} \\
-0,0041 \\
0,018 \\
\end{array}$ & $\begin{array}{l}0,029 \\
0,020 \\
0,022 \\
\end{array}$ \\
\hline $\begin{array}{l}\text { Transport } \\
\text { Logarithme du budget transport }\end{array}$ & $-0,00032$ & 0,0083 & 0,010 & 0,016 & $0,025^{* * *}$ & 0,0075 \\
\hline $\mathbf{R}^{2}$ & \multicolumn{2}{|c|}{0,47} & \multicolumn{2}{|c|}{0,43} & \multicolumn{2}{|c|}{0,19} \\
\hline Root Mean Square Error & \multicolumn{2}{|c|}{0,35} & \multicolumn{2}{|c|}{0,35} & \multicolumn{2}{|c|}{0,34} \\
\hline $\begin{array}{l}\text { Modèle } 2 \text { - Logarithme du revenu net } \\
\text { mensuel rapporté au revenu mensuel }\end{array}$ & 0,038 & 0,097 & 0,061 & 0,13 & $-0,25^{* \star *}$ & 0,084 \\
\hline $\begin{array}{l}\text { Modèle } 3 \text { - Distance domicile - travail du } \\
\text { chef de ménage (en } \mathrm{km} \text { ) }\end{array}$ & $-0,0020^{\star *}$ & 0,0010 & 0,00064 & 0,00084 & 0,0015 & 0,0010 \\
\hline $\begin{array}{l}\text { Modèle } 4 \text { - Budget temps du chef de } \\
\text { ménage (en minutes, jour de semaine) }\end{array}$ & $-0,00022$ & 0,00015 & $-0,00018$ & 0,00017 & $-0,00006$ & 0,00015 \\
\hline $\begin{array}{l}\text { Modèle } 5 \text { - Zone IAURIF de résidence } \\
\text { Zone } 1\end{array}$ & $0,13^{\star \star *}$ & 0,021 & 0,054 & 0,045 & $0,097^{\star \star *}$ & 0,026 \\
\hline Zone 2 & \multicolumn{2}{|c|}{ Réf. } & \multicolumn{2}{|c|}{ Réf. } & \multicolumn{2}{|c|}{ Réf. } \\
\hline Zone 3 & $-0,0065$ & 0,023 & $-0,050$ & 0,031 & 0,0056 & 0,021 \\
\hline Zone 4 & 0,035 & 0,039 & $-0,037$ & 0,035 & $0,071^{* *}$ & 0,034 \\
\hline Zone 5 & 0,072 & 0,046 & 0,016 & 0,036 & $0,13^{* * *}$ & 0,028 \\
\hline Zone 6 & 0,015 & 0,040 & $-0,021$ & 0,035 & $0,078^{* * *}$ & 0,030 \\
\hline Zone 7 & $-0,065$ & 0,067 & $-0,10$ & 0,074 & $-0,088$ & 0,059 \\
\hline Zone 8 & 0,094 & 0,078 & $-0,098^{* *}$ & 0,044 & $0,19^{*}$ & 0,11 \\
\hline
\end{tabular}

Lecture: régression linéaire du logarithme de la dépense de logement sur différents facteurs ayant attrait aux caractéristiques du ménage ou au coût de transport. Les coefficients sont adjoints d'astérisques s'ils sont significatifs au seuil de 1\%(***), 5\%(**), ou $10 \%(*)$.

Champ : locataires et accédants à la propriété (sauf premier sextile de revenu et revenu/budget logement non renseigné)

Source : Enquête Globale de Transport 2001-2002, DREIF

Enfin, les variables de coût de transport sont majoritairement non significatives, ou d'impact marginal. L'élasticité du budget logement au budget transport est nulle, sauf pour le secteur locatif social (avec une élasticité de 0,025). Le modèle 2 parvient à une conclusion similaire, avec cette fois un coefficient de $-0,25$ pour le logarithme du rapport entre revenus net et total. Ces signes contraires aux prédictions du modèle théorique pourraient traduire les fortes contraintes pesant lors du choix de logement social, le ménage ne pouvant choisir que parmi un nombre d'alternatives souvent très limité. Les modèles 3 ou 4 trouvent un impact nul ou marginal de la distance domicile-travail et du budget temps du chef de ménage.

Nos résultats sont basés sur une régression linéaire, méthode fournissant des effets moyens. Or les différents facteurs considérés pourraient avoir un impact différencié selon la position dans la distribution de la dépense de logement. En particulier, le coût de transport pourrait avoir un effet moyen nul sur la dépense de logement, mais impacter la queue de distribution. ${ }^{9}$

\footnotetext{
${ }^{9} \mathrm{Si}$ un ménage a un budget transport élevé, cela réduit mécaniquement sa capacité à avoir un budget logement plus élevé que la moyenne. La variable budget transport pourrait donc avoir un impact sur les quantiles correspondant à la queue supérieure de la distribution du fait de la contrainte budgétaire.
} 
Afin de confirmer la robustesse de nos résultats, nous ré-estimons donc le modèle 1 sous la forme d'une régression quantile. L'élasticité du budget logement au budget transport demeure non significative à tous les déciles pour le secteur locatif privé et les ménages accédants, et faiblement positive dans le secteur social (annexe, Graphique V). ${ }^{10}$

L'absence de fongibilité des dépenses de logement et de transport ne signifie pas une absence totale d'arbitrage entre accessibilité et coût du logement. Pour tous les statuts d'occupation, nous trouvons que le logarithme du budget transport est corrélé positivement à la superficie et négativement au coût au $\mathrm{m}^{2}$ du logement (obtenu en divisant le budget logement par la surface). L'éloignement est en moyenne bien associé à des surfaces plus grandes et à des prix immobiliers moins élevés. Cependant, tandis que le modèle théorique prédit une baisse du budget logement, on observe au contraire un budget logement inchangé avec l'éloignement. Les ménages éloignés surconsommeraient donc du logement par rapport aux prédictions du modèle théorique.

L'analyse des résidus de la régression fait également apparaître une corrélation importante des résidus avec le coût au $\mathrm{m}^{2}$. Le coefficient est de 0,55 pour le secteur locatif privé, 0,62 pour le secteur social et 0,63 pour les accédants, les trois paramètres étant significatifs au seuil de $1 \%$. Ces coefficients doivent être interprétés avec précaution du fait du problème d'endogénéité (prix et budget étant choisis de manière simultanée). La forte corrélation tend néanmoins à indiquer que le coût au $\mathrm{m}^{2}$ constituerait le second déterminant essentiel du budget logement en sus du revenu du ménage. Ceci fournit une explication aux résultats du modèle 5 . Les variables zonales sont toutes non significatives pour les locataires privés et les accédants, sauf à Paris pour le secteur locatif privé et dans les communes rurales pour l'accession. Or ces zones se distinguent par des prix au $\mathrm{m}^{2}$ particulièrement élevés (pour Paris en location) ou faibles (pour les communes rurales en accession). Les indicatrices de ces zones captureraient donc un effet prix immobilier et non un effet coût de transport. Dans les autres zones, l'hétérogénéité plus forte des prix immobiliers les rendrait exemptes de ce problème.

\section{Conclusion}

L'hypothèse de fongibilité des budgets logement et transport est donc invalidée par nos tests économétriques. Deux pistes principales se profilent pour expliquer ce résultat. Un, le modèle théorique utilisé ici pourrait mal représenter le comportement des ménages. Alternativement, les ménages pourraient méconnaître les coûts de transport. En particulier, les possesseurs de voiture pourraient mal appréhender les coûts fixes associés à l'usage de ce mode de transport, alors que ceux-ci représentent une part majeure du budget. Ces pistes feront l'objet de futurs travaux.

\section{Bibliographie}

Agenais-Gueguen A.-E. (2008), La tarification des déplacements : évaluation de la pertinence d'une évolution tarifaire pour l'Ile-de-France, doctorat de l'ENPC, février, $400 \mathrm{p}$.

Berri A. (2007), « Residential location and household expenditures on transport and housing: the example of the Greater Paris Region », Actes de la $11^{\text {ème }}$ World Conference on Transport Research, 24-28 Juin 2007 (Berkeley, USA), 57 p.

\footnotetext{
${ }^{10}$ Par souci de concision, les résultats ne sont présentés que pour le secteur locatif privé. Les autres résultats sont disponibles auprès des auteurs sur demande.
} 
Cérani N. et Camus M. (2004), «Le budget des familles en $2001 »$, Insee Résultats, $\mathrm{n}^{\circ} 29$.

CGDD (2012), «Comptes du logement: Premiers résultats 2011 - Le compte $2010 »$, Rapport de la Commission des Comptes du Logement.

Coulombel N. (2010), Toward a better representation of housing demand: On the role of monetary and non-monetary costs in household residential strategies, doctorat de l'ENPC, février, 355p.

Daubresse M. (2003), « La Reprise de l'Accession à la Propriété », Insee Première, nº 913.

DAEI/SES-P et DGUHC (2007), «Comptes du Logement 2005 et Premiers Résultats 2006 », Rapport de la Commission des Comptes du Logement.

Driant J.-C. et Jacquot A. (2005), «Loyers Imputés et Inégalités de Niveau de Vie », Economie et Statistique, ${ }^{\circ}$ 381-382, pp. 177-206

Fujita M. (1989), Urban Economic Theory: Land Use and City Size, University of Cambridge Press.

Gallot P., Leprévost E. et Rougerie C. (2011), « Prix des logements anciens et loyers entre 2000 et $2010 »$, Insee Première, $\mathrm{n}^{\circ}$ 1350, 4p.

Garcia Castello F.J. (2006), « Coût des transports en commun en la région d'Île-de-France », Document technique pour la RATP.

Gobillon L. (2001), «Emploi, logement et mobilité résidentielle », Économie et Statistique, $\mathrm{n}^{\circ}$ 349-350, pp. 77-98.

Goodman A.C. (1990), «Demographics of individual housing demand », Regional Science and Urban Economics, Vol. 20, pp. 83-102.

Haas P.M., Makarewicz C., Benedict A., Sanchez T.W. and Dawkins C.J. (2006), «Housing \& Transportation Cost Trade-Offs and Burdens of Working Households in 28 Metros », Report for the Center of Neighborhood Technology.

Hare P.H. (1995), « Clunker Mortgages and Transportation Redlining: how the mortgage banking industry unknowingly drains cities and spreads sprawl», Document de travail, Patrick H. Hare Planning and Design (Washington, DC), 21p.

Henderson J.V., Ioannides Y. (1986), «Tenure choice and the demand for housing », Economica, New Series, Vol. 53, $\mathrm{n}^{\circ} 210$, pp. 231-246.

Leurent F., Samadzad M. (2010), «Une caractérisation de la mobilité quotidienne des Franciliens, à partir de l'EGT 2001-2002 », Congrès International Atec 2010, 3-4 février, Versailles, France.

Observatoire de l'Énergie (2003), «Consommation de carburants des voitures particulières en France, 1990-2001», Document de travail.

Omalek L. (2003), «Le logement: une dépense importante pour les ménages franciliens modestes », Insee Ile-de-France à la Page, $\mathrm{n}^{\circ} 230$.

Polacchini A. and Orfeuil J-P. (1999), «Les dépenses des ménages franciliens pour le logement et les transports », Recherche Transports Sécurité, n 63, pp. 31-46. 


\section{Annexe}

Graphique II

\section{Le zonage IAURIF}

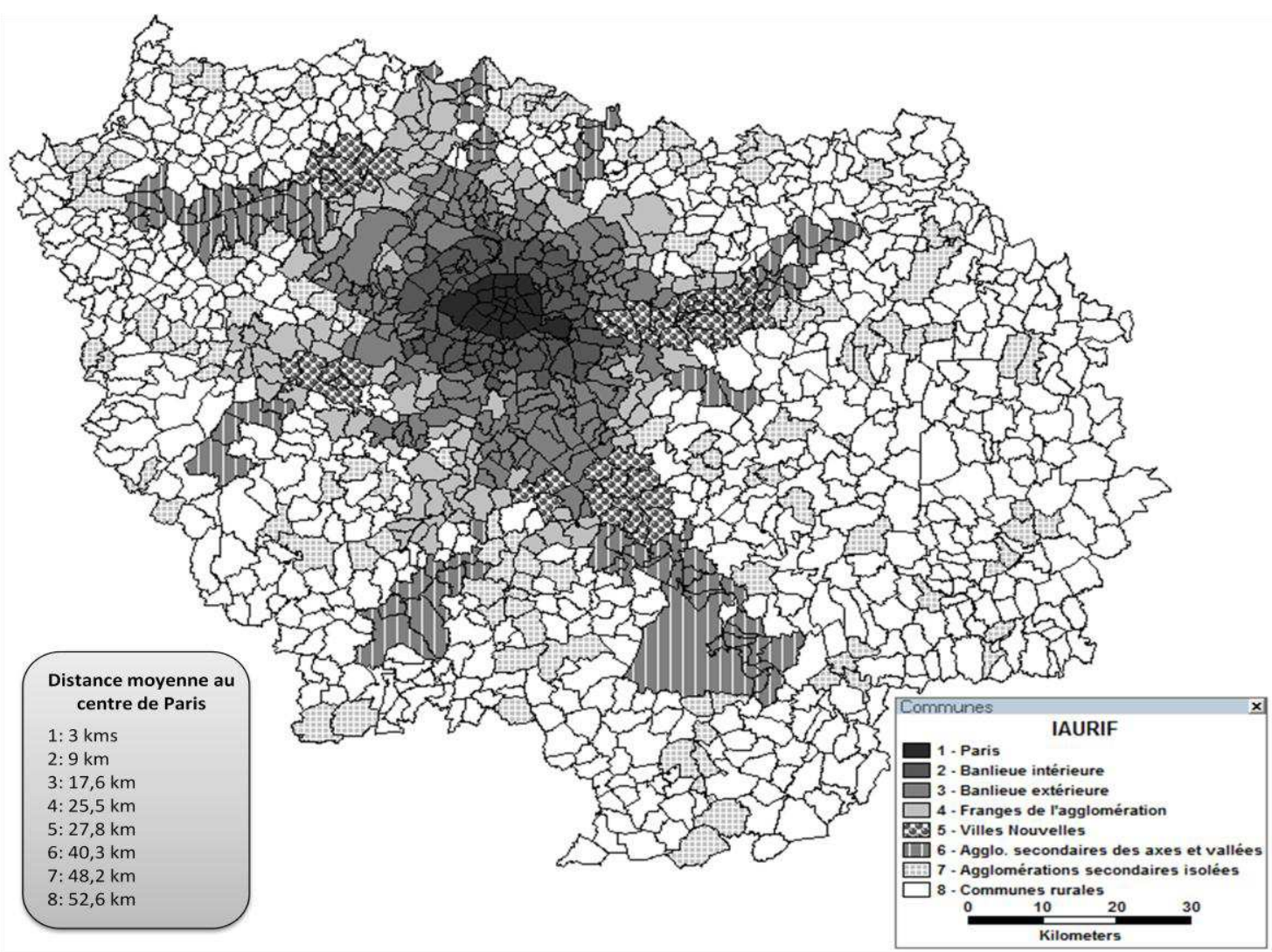

Source : IAU-IdF et calculs de l'auteur

Tableau 6

\section{Composition de l'échantillon}

\begin{tabular}{|l|c|c|c|c|c|c|c|c|c|}
\hline \multirow{2}{*}{ Zone IAURIF } & \multicolumn{3}{|c|}{ Accédants } & \multicolumn{3}{c|}{ Locatif Social } & \multicolumn{3}{c|}{ Locatif Privé } \\
\cline { 2 - 9 } & $\begin{array}{c}\text { 2ème } \\
\text { Sextile }\end{array}$ & $\begin{array}{c}\text { Tercile } \\
\text { Médian }\end{array}$ & $\begin{array}{c}\text { Tercile } \\
\text { Sup. }\end{array}$ & $\begin{array}{c}\text { 2ème } \\
\text { Sextile }\end{array}$ & $\begin{array}{c}\text { Tercile } \\
\text { Médian }\end{array}$ & $\begin{array}{c}\text { Tercile } \\
\text { Sup. }\end{array}$ & $\begin{array}{c}\text { 2ème } \\
\text { Sextile }\end{array}$ & $\begin{array}{c}\text { Tercile } \\
\text { Médian }\end{array}$ & $\begin{array}{c}\text { Tercile } \\
\text { Sup. }\end{array}$ \\
\hline Paris & 11 & 19 & 58 & 95 & 81 & 57 & 149 & 175 & 233 \\
Banlieue intérieure & 22 & 58 & 143 & 282 & 219 & 121 & 165 & 168 & 217 \\
Banlieue extérieure & 31 & 89 & 221 & 215 & 173 & 70 & 113 & 123 & 128 \\
Franges de l'agglomération & 6 & 47 & 141 & 49 & 47 & 16 & 34 & 26 & 31 \\
Villes Nouvelles & 8 & 65 & 98 & 77 & 75 & 32 & 17 & 25 & 20 \\
Villes secondaires & 12 & 74 & 101 & 83 & 60 & 18 & 33 & 25 & 29 \\
axes/vallées & & & & & & & & &
\end{tabular}




\begin{tabular}{|l|c|c|c|c|c|c|c|c|c|} 
Villes secondaires isolées & 0 & 12 & 14 & 19 & 14 & 1 & 7 & 13 & 7 \\
Communes rurales & 7 & 18 & 67 & 7 & 1 & 1 & 4 & 8 & 8 \\
\hline Ensemble & 97 & 382 & 843 & 827 & 670 & 316 & 522 & 563 & 673 \\
\hline
\end{tabular}

Lecture: la base de données contient 58 observations pour la catégorie des ménages accédants appartenant au tercile médian et résidant en banlieue intérieure.

Champ : locataires et accédants à la propriété (sauf premier sextile de revenu et revenu/budget logement non renseigné)

Source : Enquête Globale de Transport 2001-2002, DREIF

Tableau 7

Taux d'effort logement brut et net des aides personnelles selon le niveau de revenu

\begin{tabular}{|l|cc|cc|cc|}
\hline \multirow{2}{*}{ Décile de revenu } & \multicolumn{2}{|c|}{ Locatif privé } & \multicolumn{2}{c|}{ Accédants } & \multicolumn{2}{c|}{ Locatif social } \\
\cline { 2 - 7 } & $\begin{array}{c}\text { Taux effort } \\
\text { brut }\end{array}$ & $\begin{array}{c}\text { Taux effort } \\
\text { net }\end{array}$ & $\begin{array}{c}\text { Taux effort } \\
\text { brut }\end{array}$ & $\begin{array}{c}\text { Taux effort } \\
\text { net }\end{array}$ & $\begin{array}{c}\text { Taux effort } \\
\text { brut }\end{array}$ & $\begin{array}{c}\text { Taux effort } \\
\text { net }\end{array}$ \\
\hline 1 & $157 \%$ & $109 \%$ & $81 \%$ & $65 \%$ & $86 \%$ & $42 \%$ \\
3 & $50 \%$ & $38 \%$ & $59 \%$ & $55 \%$ & $32 \%$ & $22 \%$ \\
4 & $40 \%$ & $35 \%$ & $59 \%$ & $53 \%$ & $25 \%$ & $20 \%$ \\
5 & $34 \%$ & $31 \%$ & $30 \%$ & $28 \%$ & $21 \%$ & $17 \%$ \\
6 & $26 \%$ & $24 \%$ & $30 \%$ & $29 \%$ & $19 \%$ & $14 \%$ \\
7 & $24 \%$ & $23 \%$ & $28 \%$ & $26 \%$ & $16 \%$ & $14 \%$ \\
8 & $22 \%$ & $22 \%$ & $24 \%$ & $22 \%$ & $14 \%$ & $13 \%$ \\
10 & $21 \%$ & $21 \%$ & $23 \%$ & $23 \%$ & $13 \%$ & $12 \%$ \\
9 & $19 \%$ & $19 \%$ & $20 \%$ & $20 \%$ & $11 \%$ & $11 \%$ \\
\hline
\end{tabular}

Champ : locataires et accédants à la propriété, Île-de-France

Source : ENL 2002, Insee

Tableau 8

Décomposition du budget transport et niveau de motorisation des ménages franciliens

\begin{tabular}{|c|c|c|c|c|c|c|c|}
\hline \multirow[b]{2}{*}{ Zone IAURIF } & \multicolumn{3}{|c|}{ Véhicules Particuliers } & \multicolumn{2}{|c|}{ Transports en Commun } & \multirow[b]{2}{*}{ Total } & \multirow{2}{*}{$\begin{array}{r}\text { Nombre } \\
\text { moyen de } \\
\text { voitures }\end{array}$} \\
\hline & $\begin{array}{c}\text { Coûts } \\
\text { variables }\end{array}$ & $\begin{array}{l}\text { Coûts } \\
\text { fixes }\end{array}$ & Parking & Tickets & Abonnements & & \\
\hline Paris & 25 & 51 & 30 & 22 & 22 & 151 & 0,48 \\
\hline Banlieue intérieure & 50 & 85 & 24 & 15 & 21 & 195 & 0,81 \\
\hline Banlieue extérieure & 79 & 113 & 23 & 14 & 21 & 249 & 1,07 \\
\hline $\begin{array}{l}\text { Franges de } \\
\text { l'agglomération }\end{array}$ & 115 & 149 & 28 & 14 & 16 & 322 & 1,40 \\
\hline Villes Nouvelles & 106 & 126 & 23 & 16 & 26 & 296 & 1,20 \\
\hline Villes secondaires & 119 & 136 & 26 & 13 & 21 & 316 & 1,28 \\
\hline
\end{tabular}




\begin{tabular}{|l|c|c|c|c|c|c|c|} 
Villes secondaires isolées & & & & & & & \\
Communes rurales & 145 & 136 & 20 & 13 & 13 & 327 & 1,29 \\
\hline Ensemble & 225 & 207 & 53 & 30 & 16 & 531 & 1,90 \\
\hline
\end{tabular}

Lecture : un ménage résidant dans une Ville Nouvelle dispose d'1,2 voitures en moyenne. Il consacre $296 \epsilon$ par mois à sa mobilité quotidienne, dont $23 €$ en frais de stationnement.

Champ : locataires et accédants à la propriété, mobilité quotidienne

Source : Enquête Globale de Transport 2001-2002, DREIF

\section{Graphique III}

Distance parcourue moyenne selon la zone de résidence (par jour de semaine)

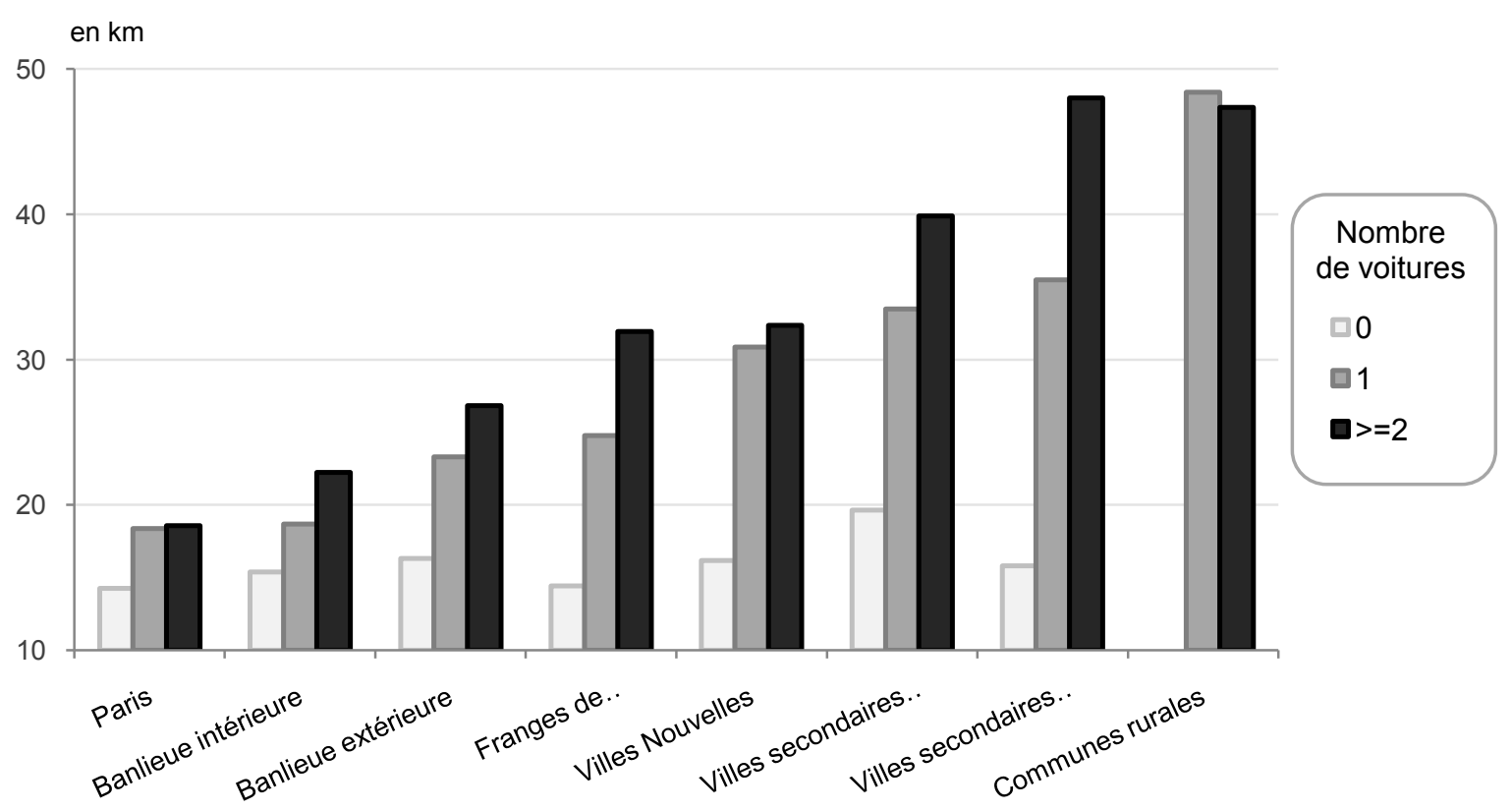

Lecture: le budget temps d'un individu vivant dans une commune rurale et dont le ménage dispose d'une seule voiture (effectif de 85 individus dans l'échantillon) était de 80,8 minutes en moyenne pour un jour de semaine.

Champ : locataires et accédants à la propriété, mobilité quotidienne Les catégories avec moins de 10 observations ne sont pas représentées.

Source : Enquête Globale de Transport 2001-2002, DREIF

Graphique IV

Temps moyen passé dans les transports selon la zone de résidence (en jour de semaine) 


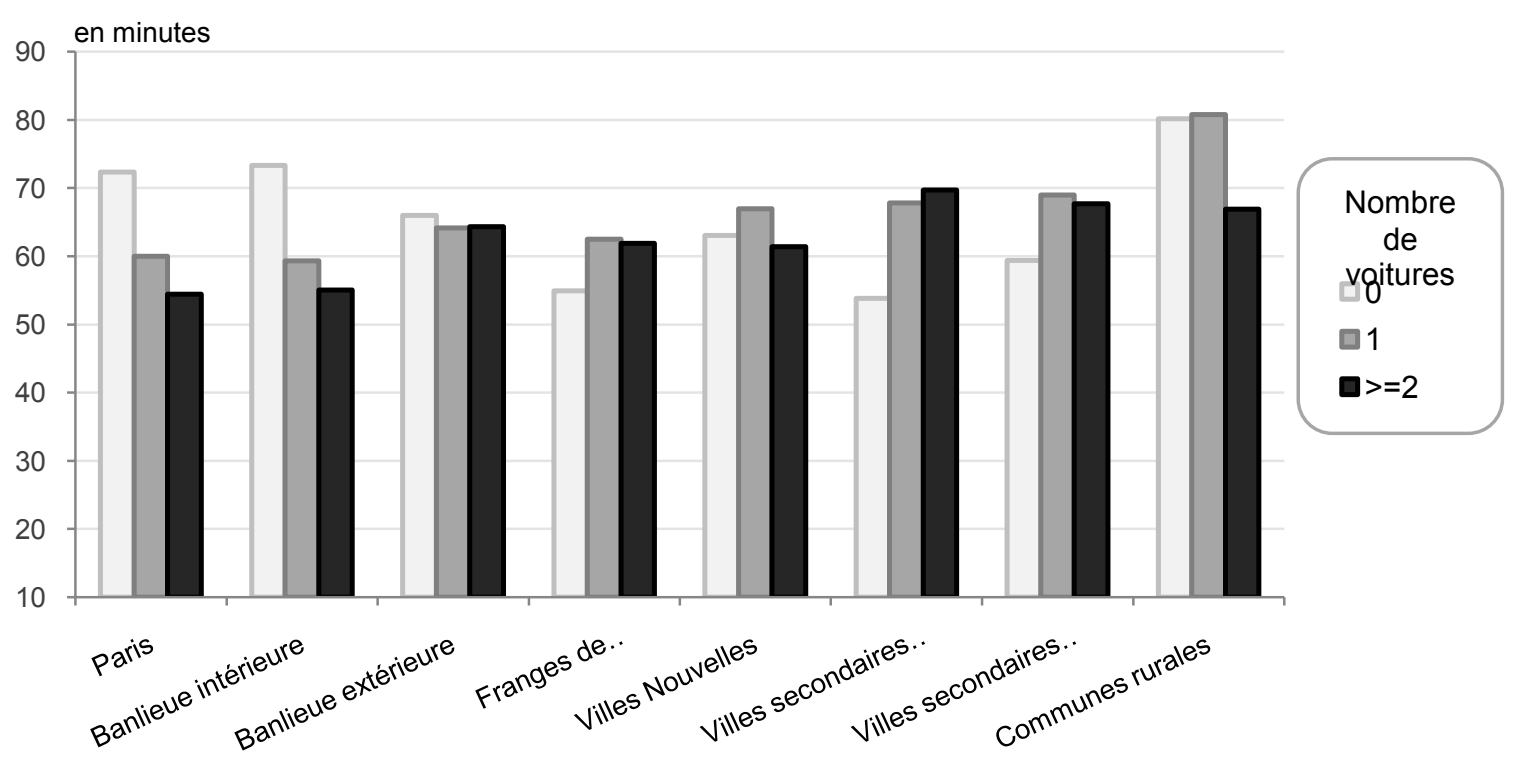

Lecture: le budget temps d'un individu vivant dans une commune rurale et dont le ménage dispose d'une seule voiture (effectif de 85 individus dans l'échantillon) était de 80,8 minutes en moyenne pour un jour de semaine.

Champ : locataires et accédants à la propriété, mobilité quotidienne

Source : Enquête Globale de Transport 2001-2002, DREIF

\section{Graphique V}

\section{Effet du budget transport sur les quantiles du budget logement}

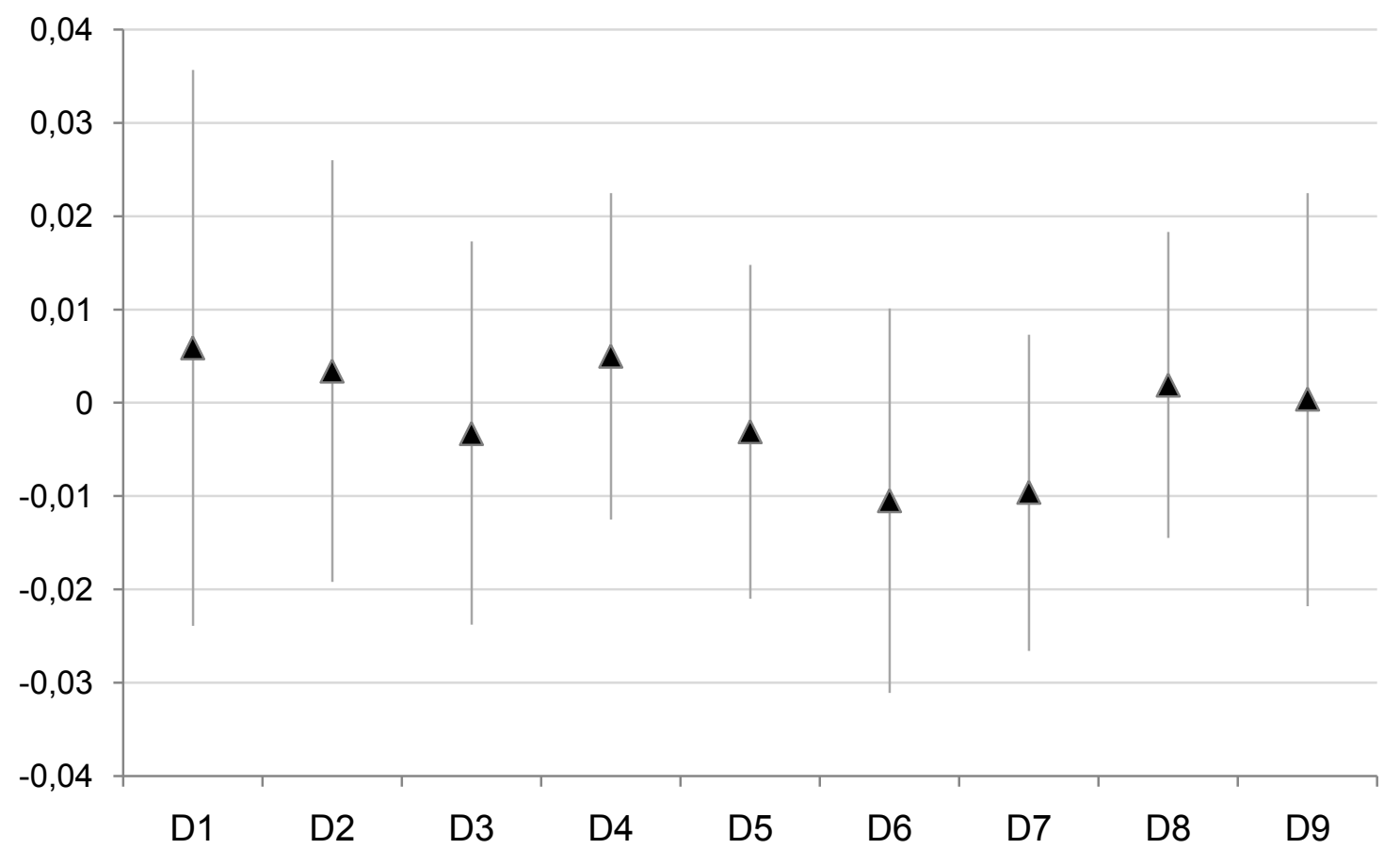

Lecture : valeur estimée et intervalle de confiance du paramètre du logarithme de la dépense de transport dans la régression quantile du logarithme de la dépense de logement. Pour le $3^{\text {ème }}$ décile, la valeur estimée est de -0,0033. L'intervalle de confiance à $95 \%$ est $[-0,0238 ; 0,0173]$.

Champ : locataires du secteur privé (sauf premier sextile de revenu et revenu/budget logement non renseigné)

Source : Enquête Globale de Transport 2001-2002, DREIF 